\title{
Preoperative and Postoperative Diffusion Tensor Imaging in Patients with Extra-Axial Lesions at the Frontal or Temporal Regions of the Brain and Their Correlations with Neuropsychological Outcomes
}

\author{
Lim Liang Hooi' ${ }^{1}$ Zamzuri Idris,2, Win Mar ${ }^{3}$, Wan Nor Azlen Wan Mohamad1 \\ ${ }^{1}$ Department of Neurosciences, School of Medical Sciences, Universiti Sains Malaysia, Kubang Kerian, Malaysia \\ ${ }^{2}$ Center for Neuroscience Service and Research, School of Medical Sciences, Universiti Sains Malaysia, \\ Kubang Kerian, Malaysia \\ ${ }^{3}$ Department of Radiology, School of Medical Sciences, Universiti Sains Malaysia, Kubang Kerian, Malaysia \\ Email: kelvin80@gmail.com
}

How to cite this paper: Hooi, L.L., Idris, Z., Mar, W. and Mohamad, W.N.A.W. (2016) Preoperative and Postoperative Diffusion Tensor Imaging in Patients with Extra-Axial Lesions at the Frontal or Temporal Regions of the Brain and Their Correlations with Neuropsychological Outcomes. J. Biomedical Science and Engineering, 9, 611-623. http://dx.doi.org/10.4236/jbise.2016.913052

Received: October 25, 2016

Accepted: December 5, 2016

Published: December 8, 2016

Copyright $\odot 2016$ by authors and Scientific Research Publishing Inc. This work is licensed under the Creative Commons Attribution International License (CC BY 4.0).

http://creativecommons.org/licenses/by/4.0/

(c) (i) Open Access

\begin{abstract}
The underlying changes in the neuronal connectivity adjacent to brain tumours cannot always be depicted by conventional MR imaging. The hypothesis of this study was that preoperative neuropsychological deficits were associated with impairment of diffusivity in association fibre bundles. Hence, we investigated the potential of combined diffusion tensor imaging (DTI) fibre tracking and fractional anisotropy (FA) values of the fibres to determine changes in association fibres and their correlation to neuropsychological scores. Our study consisted of eighteen patients with extra-axial brain tumours in areas adjacent to the frontal and temporal lobes. They were assessed pre- and postoperatively with DTI and neuropsychological assessments. MR examinations were performed on a 3T-scanner. FA values were calculated for the uncinate fasciculus, arcuate fasciculus, superior fronto-occipital fasciculus, inferior fronto-occipital fasciculus and corticospinal tracts ipsilateral and contralateral to the tumor. These values were compared with neuropsychological scores for language, memory and attention. The analysis revealed marked differences in pre- and post-excision of the tumor in both FA values and neuropsychological scores. Quantitative DTI was able to show significant differences in diffusivity of the association fibres before and after the surgery $(\mathrm{P}<0.05)$. The additional use of DTIfibre integrity and neuropsychological tests may aid in prognostication and decision making prior to surgery.
\end{abstract}




\section{Keywords}

Diffusion Tensor Imaging, Brain Tumours, Neuropsychology, Brain Mapping, Fractional Anisotropy

\section{Introduction}

Diffusion tensor imaging (DTI) involves a subset of magnetic resonance imaging that exploits the phenomenon of anisotropy in cellular microstructure to generate quantitative visual depictions of in vivo structure and function of tissue pathways [1]. This method has been widely applied in the study of white matter tracts of the brain with significant insights generated with regard to neural connections and their association with neurological disease, including intracranial tumours [2] [3] [4]. Diffusion tensor imaging in particular allows a quick, noninvasive, in vivo mapping of white matter tracts and potential patterns of impairment in function and structure. Patterns of impairment of white matter tracts in intracranial tumours have been described such as disruption, inflitration, displacement and widening [5]. Information gained from such tractographic analyses of white matter tracts have been applied clinically to determine maximal safe margins of resection of intracranial tumours with respect to adjacent white matter fibres [6].

White matter fractional anisotropy (FA) is thought to be related to white matter integrity and decline in FA is often used as an index of decreasing WM health [7]. Mean diffusivity (MD) and fractional anisotropy (FA) are the two most commonly used measures of white matter integrity. FA refers to the coherence of the orientation of water diffusion, independent of rate. It can be calculated as the fraction of total diffusion that can be attributed to anisotropic diffusion, which is derived from the normalized variances of the three eigenvalues [8], with higher values corresponding to a more consistent diffusion orientation. Taken together, a breakdown in white matter integrity would be seen as lower FA [9].

The frontal and temporal lobes have long to be known to play a vital role in speech, memory and attention of normal individuals, more so the dominant hemisphere [10] [11] [12] [13].

Our study is an attempt to quantify the neurologic dysfunction that results from the impairment of major association white matter tracts as defined via diffusion tensor imaging with a standardized neuropsychological assessment, specifically in patients with extra-axial tumours. The utility of neuropsychological assessment in this study lies in the identification of potential deficits that would not be easily acquired via routine bedside neurological examination. Here, we present our results on eighteen patients with intracranial extra-axial tumours located at the frontal or temporal lobes and analyzed their neuropsychological scores for memory, attention and language in relation to the fractional anisotropy values (FA) for uncinate fasciculus (UF), arcuate fasciculus (AF), corticospinal tract (CST), inferior fronto-occipital fasciculus (IFOF) and superior fron- 
to-occipital fasciculus (SFOF).

\section{Materials and Methods}

\subsection{Subjects}

Eighteen patients with extra-axial brain tumours adjacent to the frontal and temporal lobes were assessed pre- and postoperatively with DTI and neuropsychological assessments. The first patient was recruited in January 2014 and the trial was closed to recruitment in November 2015. The preoperative assessments were done on initial diagnosis prior to starting the patient on steroids. The postoperative assessments were done at 6 weeks after the surgery. The study was approved by the local research and ethics committee (ref: 304/PPSP/61312142) and informed consent was obtained from all subjects. Patients recruited were adults over the age of 12 to 70 years, with extra-axial lesions at the frontal and temporal regions of the brain, and with valid consent from the patient or direct family members if the patient was unable to give informed consent. Patients who did not fit into the age range, and with existing conditions which could independently affect the outcome scores, or who were not suitable for surgery or MRI were not included into the study. From the literature review for DTI, the significant detectable difference for pre and postoperative data from previous study for DTI FA values $(\delta)$ was 0.04 [7] and the standard deviation $(\sigma)$ for normal control was 0.06 . Therefore, the calculated sample size using t-test comparing 2 means formula was 20 . Considering 20\% drops from the study, the total required number of patient was 24 . However due to time constraints and limited number of patients with matching pathology, we only managed to recruit 18 patients.

\subsection{DTI Study}

All MR examinations were performed on a 3T-MRI scanner (Philips Achieva). Mean FA values from three readings were calculated for uncinate fasciculus (UF), arcuate fasciculus (AF), corticospinal tract (CST), inferior fronto-occipital fasciculus (IFOF) and superior fronto-occipital fasciculus (SFOF) (Figure 1) in both cerebral hemispheres. These values were compared with neuropsychological scores for memory, language, and attention.

\subsection{Data Acquisition}

DTI was performed on the whole brain with the following parameters: single shot spin echo planar imaging with $\mathrm{TE}=69 \mathrm{~ms}, \mathrm{TR}=10,190 \mathrm{~ms}$, axial slices, SENSE factor 3.2, number of signals averaged 6 , half scan factor 0.712 , isotropic $2.2 \mathrm{~mm} \times 2.2 \mathrm{~mm} \times 2.2$ $\mathrm{mm}$ voxels, $\mathrm{b}=0 \mathrm{~s} / \mathrm{mm}^{2}$ plus 15 diffusion-sensitizing gradient directions $(\mathrm{b}=800$ $\mathrm{s} / \mathrm{mm}^{2}$ ) and phase encoding in the anterior-posterior direction. Total scan time was 16 min. A T1-weighted scan (3D T1-TFE) was performed as an anamical reference using identical orientation and slice thickness as the DTI scan. Reconstructed pixel size was $1.9 \mathrm{~mm} \times 1.9 \mathrm{~mm}$ for both DTI and T1-weighted images. 


\subsection{Data Processing}

DTI data was acquired using the "FiberTrak" package included in the Extended MRI Workspace (EWS) R 2.6.3 (Philips Achieva 3.0T X-series). The DTI data was transferred to a workstation (8 GB RAM, $2.33 \mathrm{GHz}$ Intel Xeon, Windows 64 bit). This fibre tracking software is based on the "fibre assignment by continuous tracking" (FACT) algorithm. To minimize artifacts caused by movement and eddy currents, coregistration to $\mathrm{b}=0$ scans was done using the diffusion registration package in the EWS. Fractional anisotropy maps were generated in the EWS. Settings for the tractography were the following: $\mathrm{FA}>0.25$, maximal angle change $90^{\circ}$, minimal fibre length $30 \mathrm{~mm}$. Regions of interests (ROIs) were selected to measure the FA values in both hemispheres. The uncinate fasciculus was identified as a white matter tract connecting the medial basal pole of the temporal lobe with the inferolateral part of the basal frontal lobe. The arcuate fasciculus was easily identified at the terminal end of the sylvian fissure deep into the white matter. The corticospinal tract is the fibre tract that runs from the motor cortex (anterior to the central sulcus) down to the posterior limb of the internal capsule. The inferior fronto-occipital fasciculus is the white matter tract that connects the frontal lobe with the occipital lobe and passes through the inner aspect of the insular cortex whilst the superior fronto-oocipital fasciculus was identified as a white matter tract that connects the frontal with the occipital lobe at the medial and superior aspect of the corticospinal tract [14] [15] [16]. These tracts are demonstrated in Figure 1. Figure 2 shows a pre- and post-operative MRI and DTI with fibre tracts demonstrated.

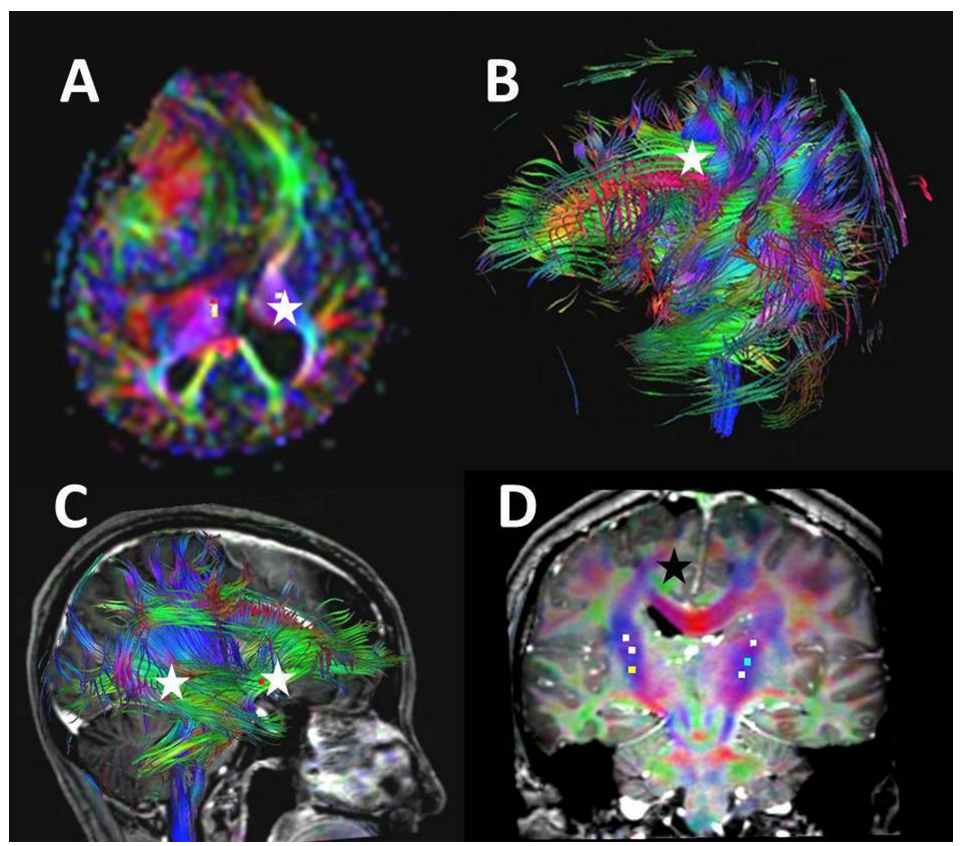

Figure 1. The studied white matter tracts are identified using FA map and DTI fibre-tracking. (A) The white star indicates the corticospinal tract; (B) The white star indicates the arcuate fasciculus; (C) The white star anteriorly located signifies the uncinate fasciculus whilst the posterior one indicates the inferior fronto-occipital fasciculus; and (D) The black star indicates the superior fronto-occipital fasciculus. 


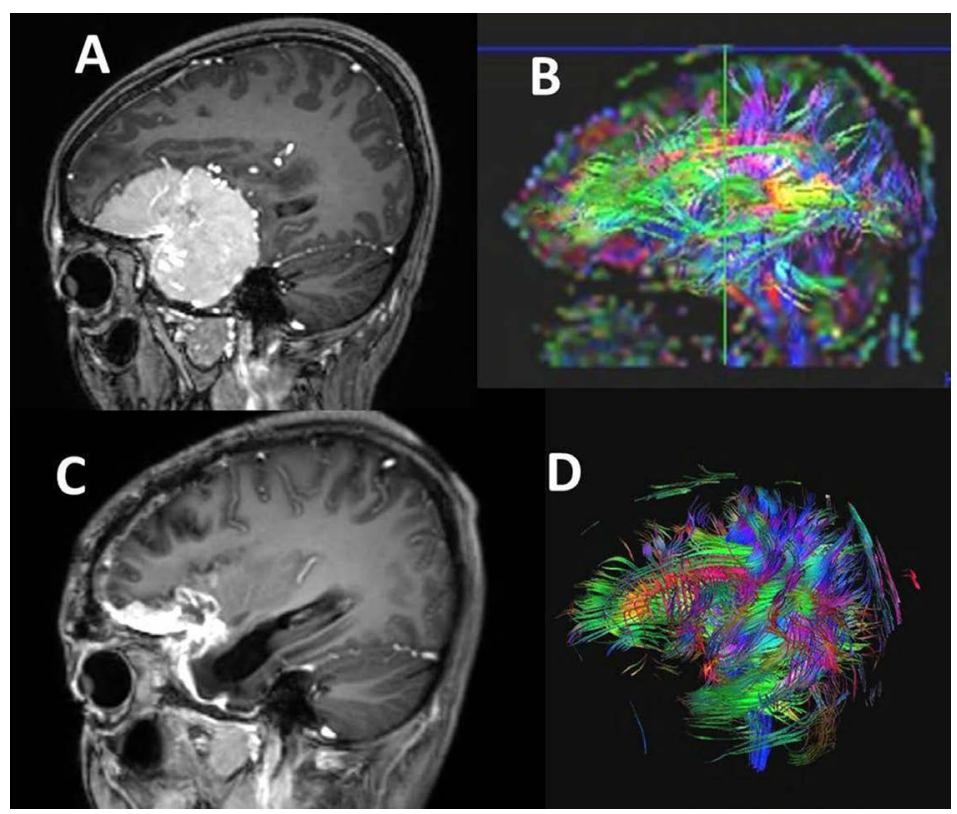

Figure 2. MRI and Diffusion Tensor Imaging (DTI) for one of the studied patients. (A) and (B) MRI and DTI prior to surgery and (C) and (D), the MRI and DTI after the surgery. Marked improvements are noted in anatomical as well as in tensor strength.

The images are then broken down into axial, sagittal and coronal planes to study the individual tracts.

\subsection{Neuropsychological Tests}

The neuropsychological tests applied for this study are divided into 3 main components: memory, attention and language assessments. The memory tests were for the immediate, delayed, auditory and total memory. The attention test used was Comprehensive Trail Making Test (CTMT) and for the language assessments, the batteries of tests were for visual naming, sentence repetition, controlled oral word association, oral spelling, written spelling, block spelling, token test, aural and reading comprehension [17] [18].

\subsection{Statistical Method}

Inferential Statistical analysis was performed with the use of commercially available software SPSS Inc. Version 20. Probability values $<0.05$ were considered significant. Analysis based on Wilcoxon-signed rank test (non-parametric test) for the significant difference between pre and post results ( $\mathrm{p}$-value $<0.05$ ).

\section{Results}

\subsection{Demographic Data of the Study}

Eighteen patient's data were analyzed for demography, clinical condition, neuropsychological impairments, and the FA values of the white matter tracts. There were 11 female and seven male patients with age ranging from 22 to 64 years of age (mean age 
of 47.22 years) of which only one was left handed. Sixteen of these patients had a tumour compressing on the left side of the brain, and the remaining 2 patients had a tumour on the right. All tumours were successfully removed with Simpson's grading of 1 or 2 with resolution of symptoms in all patients after the surgery (Simpson Grading for meningiomas surgery: Grade I-complete removal including resection of underlying bone and associated dura; Grade II-complete removal and coagulation of dural attachment; Grade III-complete removal without resection of dura or coagulation; Grade IV - subtotal resection) [19]. Further analysis was made for neuropsychological scores together with fractional anisotropy values for all studied-white matter tracts in these eighteen patients and is depicted below in Table 1.

\subsection{Component of Neuropsychological Outcomes and FA Values}

Table 2 shows the results in all the 18 parameters measured during neuropsychological assessment before and after surgery for 18 parameters tested, with p-value $<0.05$ statistically significant. The patients show a statistically significant improvement in all the parameters tested following surgery.

Table 1. Summary of the patients' demographic data detailing the age, sex, tumour side, handedness, Simpson grading, and clinical outcome of the patients.

\begin{tabular}{|c|c|c|c|c|c|c|}
\hline Case & Age & Sex & Tumor Side & Handedness & $\begin{array}{l}\text { Simpson } \\
\text { Grading }\end{array}$ & $\begin{array}{c}\text { Neuropsychological } \\
\text { Outcomes }\end{array}$ \\
\hline 1 & 48 & $\mathrm{~F}$ & $\mathrm{~L}$ & $\mathrm{R}$ & I & $\mathrm{Y}$ \\
\hline 2 & 56 & $\mathrm{~F}$ & $\mathrm{~L}$ & $\mathrm{R}$ & II & Y \\
\hline 3 & 32 & M & $\mathrm{R}$ & $\mathrm{R}$ & II & $\mathrm{Y}$ \\
\hline 4 & 46 & M & $\mathrm{L}$ & $\mathrm{R}$ & I & Y \\
\hline 5 & 53 & M & $\mathrm{L}$ & $\mathrm{R}$ & II & Y \\
\hline 6 & 26 & $\mathrm{~F}$ & $\mathrm{~L}$ & $\mathrm{R}$ & II & Y \\
\hline 7 & 22 & $\mathrm{~F}$ & $\mathrm{~L}$ & $\mathrm{R}$ & I & Y \\
\hline 8 & 32 & M & $\mathrm{R}$ & $\mathrm{R}$ & I & Y \\
\hline 9 & 57 & M & $\mathrm{L}$ & $\mathrm{R}$ & II & Y \\
\hline 10 & 55 & M & $\mathrm{L}$ & $\mathrm{R}$ & I & Y \\
\hline 11 & 63 & M & $\mathrm{L}$ & $\mathrm{R}$ & II & $\mathrm{Y}$ \\
\hline 12 & 51 & $\mathrm{~F}$ & $\mathrm{~L}$ & $\mathrm{~L}$ & II & Y \\
\hline 13 & 24 & $\mathrm{~F}$ & $\mathrm{~L}$ & $\mathrm{R}$ & I & Y \\
\hline 14 & 57 & $\mathrm{~F}$ & $\mathrm{~L}$ & $\mathrm{R}$ & II & Y \\
\hline 15 & 49 & $\mathrm{~F}$ & $\mathrm{~L}$ & $\mathrm{R}$ & I & Y \\
\hline 16 & 53 & $\mathrm{~F}$ & $\mathrm{~L}$ & $\mathrm{R}$ & II & Y \\
\hline 17 & 62 & $\mathrm{~F}$ & $\mathrm{~L}$ & $\mathrm{R}$ & II & $\mathrm{Y}$ \\
\hline 18 & 64 & $\mathrm{~F}$ & $\mathrm{~L}$ & $\mathrm{R}$ & II & $\mathrm{Y}$ \\
\hline
\end{tabular}

Age in years; F-Female; $\mathrm{M}-$ Male; $\mathrm{L}-$ Left; $\mathrm{R}-$ Right; $\mathrm{Y}$-Improvement in Neuropsychology scores; N-No improvement in Neuropsychology scores. 
Table 2. Pre and post-operative neuropsychological assessments analysis.

\begin{tabular}{|c|c|c|c|c|c|}
\hline \multirow{2}{*}{ Measures } & \multicolumn{2}{|c|}{ Pre } & \multicolumn{2}{|c|}{ Post } & \multirow{2}{*}{${ }^{\wedge} \mathrm{p}$-value } \\
\hline & Mean & SD & Mean & SD & \\
\hline Full-2 IQ & 75.56 & 17.212 & 86.11 & 18.026 & $0.002^{*}$ \\
\hline Immediate Memory & 70.44 & 17.002 & 82.56 & 19.954 & $0.001^{*}$ \\
\hline Delayed Memory & 72.00 & 17.974 & 84.67 & 20.243 & $0.000^{*}$ \\
\hline Total Memory & 70.28 & 16.552 & 84.39 & 18.092 & $0.000^{*}$ \\
\hline Visual Memory Correct Score & 4.17 & 2.956 & 6.83 & 2.662 & $0.001^{*}$ \\
\hline Visual Memory Error Score & 8.94 & 6.197 & 4.94 & 4.659 & $0.000^{*}$ \\
\hline CTMT Executive Function & 21.13 & 6.109 & 24.13 & 4.031 & $0.022^{*}$ \\
\hline Auditory Immediate Recall & 30.33 & 16.866 & 41.56 & 17.113 & $0.000^{*}$ \\
\hline Auditory Delayed Recall & 10.11 & 9.952 & 17.17 & 8.291 & $0.001^{*}$ \\
\hline Visual Naming & 34.76 & 12.357 & 46.39 & 7.237 & $0.000^{*}$ \\
\hline Sentence Repetition & 7.00 & 3.354 & 9.00 & 3.010 & $0.032^{*}$ \\
\hline Controlled Oral Word Association & 26.53 & 15.855 & 31.56 & 10.929 & $0.046^{*}$ \\
\hline Oral Spelling & 8.35 & 3.499 & 10.00 & 3.029 & $0.004^{*}$ \\
\hline Written Spelling & 8.53 & 3.573 & 10.00 & 2.870 & $0.012^{*}$ \\
\hline Block Spelling & 7.76 & 3.784 & 9.33 & 3.464 & $0.010^{*}$ \\
\hline Token Test & 32.88 & 12.232 & 38.50 & 8.403 & $0.001^{*}$ \\
\hline Aural Comprehension & 13.47 & 4.989 & 16.22 & 1.957 & $0.005^{*}$ \\
\hline Reading Comprehension & 14.41 & 5.767 & 17.17 & 2.684 & $0.002^{*}$ \\
\hline
\end{tabular}

$\wedge$ Analysis based on Wilcoxon-signed rank test (non-parametric test). ${ }^{*}$ Significant difference between pre and post results (p-value $<0.05)$.

\subsection{FA Values for the Pre and Postoperative White Matter Tracts}

As shown in Table 3 all measurements for the tracts in the left hemisphere demonstrated improvement and statistically significant results with $\mathrm{p}$-value $<0.05$, however only one out of the five tracts in the right cerebral hemisphere demonstrated significant difference shown on the inferior occipto-temporal (right) with p-value $=0.016$, and the remaining parameters such as uncinate tract (right), arcuate fasciculus (right), corticospinal tract (right) and superior fronto-occipital (right) with p-value $>0.05$. This could be due to a majority of the tumours presenting on the left, and thus causing the tracts in the left hemisphere to be more significantly affected while having minimal effects on the tracts in the right hemisphere. From the analysis we can surmise that there were statistically significant improvements in FA values of the white matter tracts which represent the integrity of the white matter fibres following removal of the tumour. There was also a statistically significant improvement in the neuropsychological scores for these patients. These changes seemed to contribute towards clinical as well as neuropsychological improvements in memory, attention and language. This may indirectly suggest that the brain networks for the whole brain are returning to a healthy state. 


\subsection{FA Lobes and Outcomes}

FA values of the lobes of the brain were also measured as shown in Table 4. The FA values of the right and left frontal, parietal, temporal (medial and lateral) and occipital lobes were derived, where the results show statistically significant improvement for both right and left lobes with p-value $<0.05$ for all 18 subjects. Frontal, parietal, temporal, and occipital lobe regions were defined so as to be consistent with the automated anatomic labeling (AAL) structures defined by Tzourio-Mozoyer et al. (2001) and further defined by Grieve et al. (2007) [20] [21].

Table 3. Pre- and post operative white matter tract results, FA values of 5 tracts from both cerebral hemispheres have been measured.

\begin{tabular}{cccccc}
\hline \multirow{2}{*}{ Measures } & \multicolumn{2}{c}{ Pre } & \multicolumn{2}{c}{ Post } & \multirow{2}{*}{${ }^{\wedge}$ p-value } \\
\cline { 2 - 5 } & Mean & SD & Mean & SD & \\
\hline Uncinate tract (right) & 0.519 & 0.112 & 0.536 & 0.115 & 0.053 \\
Uncinate tract (left) & 0.416 & 0.072 & 0.443 & 0.069 & $0.003^{*}$ \\
Arcuate fasciculus (right) & 0.627 & 0.048 & 0.641 & 0.035 & 0.102 \\
Arcuate fasciculus (left) & 0.484 & 0.164 & 0.524 & 0.164 & $0.003^{*}$ \\
Corticospinal tract (right) & 0.698 & 0.131 & 0.696 & 0.106 & 0.332 \\
Corticospinal tract (left) & 0.603 & 0.142 & 0.627 & 0.136 & $0.017^{*}$ \\
inferior fronto-occipital (right) & 0.722 & 0.132 & 0.745 & 0.147 & $0.016^{*}$ \\
inferior fronto-occipital (left) & 0.605 & 0.110 & 0.690 & 0.104 & $0.005^{*}$ \\
Superior fronto-occipital (right) & 0.616 & 0.068 & 0.625 & 0.106 & 0.072 \\
Superior fronto-occipital (left) & 0.452 & 0.205 & 0.513 & 0.142 & $0.007^{*}$ \\
\hline
\end{tabular}

$\wedge$ Analysis based on Wilcoxon-signed rank test (non-parametric test). ${ }^{\star}$ Significant difference between pre and post results (p-value $<0.05)$.

Table 4. Pre and post-operative FA values as defined by the different lobes.

\begin{tabular}{|c|c|c|c|c|c|}
\hline \multirow{2}{*}{ Measures } & \multicolumn{2}{|c|}{ Pre } & \multicolumn{2}{|c|}{ Post } & \multirow{2}{*}{${ }^{\wedge} \mathrm{p}$-value } \\
\hline & Mean & SD & Mean & SD & \\
\hline Right frontal & 0.335 & 0.094 & 0.347 & 0.094 & $0.000^{*}$ \\
\hline Right parietal & 0.313 & 0.065 & 0.330 & 0.070 & $0.006^{*}$ \\
\hline Right temporal (medial) & 0.286 & 0.102 & 0.316 & 0.111 & $0.010^{\star}$ \\
\hline Right temporal (lateral) & 0.302 & 0.086 & 0.334 & 0.084 & $0.000^{*}$ \\
\hline Right occipital & 0.287 & 0.045 & 0.303 & 0.049 & $0.029^{*}$ \\
\hline Left frontal & 0.340 & 0.084 & 0.361 & 0.085 & $0.003^{*}$ \\
\hline Left parietal & 0.346 & 0.095 & 0.375 & 0.103 & $0.012^{*}$ \\
\hline Left temporal (medial) & 0.344 & 0.056 & 0.366 & 0.095 & $0.004^{*}$ \\
\hline Left temporal (lateral) & 0.334 & 0.072 & 0.357 & 0.087 & $0.006^{*}$ \\
\hline Left occipital & 0.326 & 0.065 & 0.350 & 0.083 & $0.023^{\star}$ \\
\hline
\end{tabular}

$\wedge$ Analysis based on Wilcoxon-signed rank test (non-parametric test). ${ }^{\star}$ Significant difference between pre and post results (p-value $<0.05)$. 


\section{Discussion}

\subsection{Clinical Outcomes and White Matter Tracts}

For our study we explored the effect of main association white matter tracts and cognitive performance in patients with tumours compressing on the frontal or temporal lobes of the brain. We chose ROI-based analyses because of its superior validity compared to voxel-based or deformation based approaches at present. We focused on the long association fibre tracts because they represent important connections of the cortico-cortical and limbic-association cortical networks which are known to play a major role in cognitive functioning and language [22]. The fibre tracts integrity was quantified by anisotropy measurements within the arcuate fasciculus, uncinate fasciculus, superior fronto-occipital fasciculus and inferior fronto-occipital fasciculus. Muller et al. previously demonstrated a normal distribution as well as a fairly good reliability and precision of ROI-based DTI measurements using FA values [23].

We assessed eighteen patients in this study, and as shown in Table 1 there was a fairly wide age distribution among the patients (22 - 64 years), while there was slight female preponderance ( 11 female vs 7 male). The majority of the patients were right handed (17 vs 1 ) while most of the tumours were left sided (16 vs 2 ). They all underwent a craniotomy and we managed to achieve tumour excision Simpson grade I and II in all of our patients studied, and for the duration of the study none of them required a second surgery. All of the patients had shown improvement after the surgery as evidenced by the improvement in neuropsychological scoring assessments.

\subsection{Extra-Axial Tumours and White Matter Tracts-A Human Model to Study Neurocognition}

Our study demonstrates that changes in white matter tracts due to tumoral lesions are associated with detectable neuropsychological deficits, and are reversible with surgical removal of such lesions. The selection of frontal and temporal mass lesions in our study is critical due to their likely adjacence to presumed important association white matter fibres involved in speech, memory, and attention. Extra-axial mass lesions were specifically analysed due to their tendency to displace rather than infiltrate or disrupt adjacent white matter tracts [24] [25] [26]. Though the prognostic implications of different white matter tractographic abnormalities remain uncertain [27] [28], we speculate that the likelihood of postoperative resolution of neuropsychological deficits is higher with displaced fibres. The relatively rapid improvement in documented deficits as seen in the postoperative assessment of all three patients suggests that the predominant mechanism of dysfunction in such patients is white matter compression. This highlights the usefulness of this type of pathology as a model to study the effects of intracranial lesions on neuropsychology and in assessing the validity of certain neuropsychological batteries.

\subsection{Tracts for Memory, Attention and Language and Neuroplasticity}

Some patients may present with gross memory, attention or language deficits, however 
subtle cognitive deficits may sometimes only be noted via a thorough neuropsychological assessment and scored accordingly. The scoring system allows accurate comparisons to be made before and after the surgery, or after any sort of intervention. Our study confirmed the importance of doing neuropsychological tests for patients with brain tumours, particularly those affecting the frontal and temporal lobes as they can be used as an outcome assessment for the surgery as evidenced in Tables 2-4. Additional data from white matter tracts FA values in these patients further elucidated the systematic networks for all cognitive brain functions. From our data obtained from these patients, we can conclude that uncinate, arcuate and inferior fronto-occipital fasciculus in both hemispheres play significant roles in memory and language, whilst the superior fronto-occipital fasciculus may have a role in attentional network. These cognitive networks seem to be bilateral and any tract dysfunction in one brain hemisphere may cause neuroplasticity to occur in a similar tract but in the opposite hemisphere. Neuroplasticity is a constant process allowing all-time rewiring and reshaping of the neuronosynaptic maps, the purpose of this mechanism is to optimize the brain processing and network functions [29] [30]. The capacity for the brain to reorganize itself is critical for the process of functional recovery in patients, and this is often called post-lesional plasticity [31].

\subsection{FA Values for the Brain Lobes and Outcomes}

Even FA values by lobes showed significant improvement in all the lobes measured after surgery. Of note, even the occipital lobe which was not directly compressed by tumours in this study demonstrated a significant increase in FA values after surgery. This may be due to the fact that the association tracts that pass to the occipital lobe may have been affected by the tumour compression, and hence the FA values of the tract improved after surgery, rendering an overall improvement in FA values of the lobe.

\subsection{Limitations and Further Study}

There were limitations to this study such as the small sample size, and also the short period for reassessment at 6 weeks. There are also shortcomings in the assessment of white matter tracts with regions of interest being observer dependant and may be imprecise. These limitations were dealt with by assessment of regions of interest by 2 independent observers. A future study would likely benefit from a larger sample size and a longer follow up reassessment period.

\section{Conclusion}

Quantitative DTI was able to show significant differences in diffusivity of the association fibres before and after the tumoral surgery. The additional use of neuropsychological assessments with white matter fibre integrity evaluated using FA values may aid in prognostication and decision making prior to surgery, which should lead to an improved outcome for the patient in clinical as well as neuropsychological aspects. Further studies may be needed to show a correlation between the two parameters, but this 
study has shown that there are improvements in fibre integrity and neuropsychological scores after tumour removal surgery. We should pay great heed to the potential damaging effects of brain tumours and its treatment, and further studies on this subject may enable us to understand more about the brain and its functions in order to preserve it for the best possible outcome in patients.

\section{Acknowledgements}

This study was supported by short term grants (ref: 304/PPSP/61312142) from Universiti Sains Malaysia (USM), and approved by Human Research Ethics Committee USM, School of Medical Sciences, Kubang Kerian, Kelantan, Malaysia.

\section{References}

[1] Stadlbauer, A., Nimsky, C., Gruber, S., Moser, E., Hammen, T., Engelhorn, T., et al. (2007) Changes in Fiber Integrity, Diffusivity, and Metabolism of the Pyramidal Tract Adjacent to Gliomas: A Quantitative Diffusion Tensor Fiber Tracking and MR Spectroscopic Imaqinq Study. American Journal of Neuroradiology, 28, 462-469.

[2] Jiang, R., Du, F.-Z., He, C., Gu, M., Ke, Z.-W. and Li, J.-H. (2014) The Value of Diffusion Tensor Imaging in Differentiating High-Grade Gliomas from Brain Metastases: A Systematic Review and Meta-Analysis. PLoS ONE, 9, e112550.

https://doi.org/10.1371/journal.pone.0112550

[3] De Belder, F.E., Oot, A.R., Van Hecke, W., Venstermans, C., Menovsky, T., Van Marck, V., et al. (2012) Diffusion Tensor Imaging Provides an Insight into the Microstructure of Meningiomas, High-Grade Gliomas, and Peritumoral Edema. Journal of Computer Assisted Tomography, 36, 577-582. https://doi.org/10.1097/RCT.0b013e318261e913

[4] Inano, R., Oishi, N., Kunieda, T., Arakawa, Y., Yamao, Y., Shibata, S., et al. (2014) Voxel-Based Clustered Imaging by Multiparameter Diffusion Tensor Images for Glioma Grading. NeuroImage: Clinical, 5, 396-407.

[5] Morita, N., Wang, S., Kadakia, P., Chawla, S., Poptani, H. and Melhem, E.R. (2011) Diffusion Tensor Imaging of the Corticospinal Tract in Patients with Brain Neoplasms. Magnetic Resonance in Medical Sciences, 10, 239-243. https://doi.org/10.2463/mrms.10.239

[6] Yen, P.S., Teo, B.T., Chiu, C.H., Chen, S.C., Chiu, T.L. and Su, C.F. (2009) White Matter Tract Involvement in Brain Tumors: A Diffusion Tensor Imaging Analysis. Surgical Neurology, 72, 464-469. http://www.ncbi.nlm.nih.gov/pubmed/19608227

[7] Kochunov, P., Thompson, P.M., Lancaster, J.L., Bartzokis, G., Smith, S., Coyle, T., et al. (2007) Relationship between White Matter Fractional Anisotropy and Other Indices of Cerebral Health in Normal Aging: Tract-Based Spatial Statistics Study of Aging. Neuroimage, 35, 478-487. https://doi.org/10.1016/j.neuroimage.2006.12.021

[8] Basser, P.J. and Pierpaoli, C. (2011) Microstructural and Physiological Features of Tissues Elucidated by Quantitative-Diffusion-Tensor MRI. Journal of Magnetic Resonance, 213, 560-570. http://www.sciencedirect.com/science/article/pii/S109078071100334X https://doi.org/10.1016/j.jmr.2011.09.022

[9] Bennett, I., Madden, D. and Vaidya, C. (2010) Age Related Differences in Multiple Measures of White Matter Integrity: A Diffusion Tensor Imaging Study of Healthy Aging. $\mathrm{Hu}$ man Brain, 31, 378-390. http://onlinelibrary.wiley.com/doi/10.1002/hbm.20872/full

[10] Jonides, J., Lacey, S.C. and Nee, D.E. (2005) Processes of Working Memory in Mind and Brain. Current Directions in Psychological Science, 14, 2-5. 
https://doi.org/10.1111/j.0963-7214.2005.00323.x

[11] Troyer, A.K., Moscovitch, M., Winocur, G., Alexander, M.P. and Stuss, D. (1998) Clustering and Switching on Verbal Fluency: The Effects of Focal Frontal- and Temporal-Lobe Lesions. Neuropsychologia, 36, 499-504. https://doi.org/10.1016/S0028-3932(97)00152-8

[12] Crinion, J.T., Lambon-Ralph, M.A., Warburton, E.A., Howard, D. and Wise, R.J.S. (2003) Temporal Lobe Regions Engaged during Normal Speech Comprehension. Brain, 126, 11931201. https://doi.org/10.1093/brain/awg104

[13] Matthews, B.R. (2015) Memory Dysfunction. CONTINUUM: Lifelong Learning in Neurology, 21, 613-626. https://doi.org/10.1212/01.CON.0000466656.59413.29

[14] Wakana, S., Jiang, H., Nagae-Poetscher, L.M., van Zijl, P.C.M. and Mori, S. (2004) Fiber Tract-Based Atlas of Human White Matter Anatomy. Radiology, 230, 77-87. http://www.ncbi.nlm.nih.gov/pubmed/14645885 https://doi.org/10.1148/radiol.2301021640

[15] Jellison, B.J., Field, A.S., Medow, J., Lazar, M., Salamat, M.S. and Alexander, A.L. (2004) Diffusion Tensor Imaging of Cerebral White Matter: A Pictorial Review of Physics, Fiber Tract Anatomy, and Tumor Imaging Patterns. American Journal of Neuroradiology, 25, 356-369.

[16] Mori, S. and van Zijl, P.C.M. (2002) Fiber Tracking: Principles and Strategies-A Technical Review. NMR in Biomedicine, 15, 468-480. http://doi.wiley.com/10.1002/nbm.781 https://doi.org/10.1002/nbm.781

[17] Harvey, P.D. (2012) Clinical Applications of Neuropsychological Assessment. Dialogues in Clinical Neuroscience, 14, 91-99.

[18] Moses, J. (2004) Comprehensive Trail Making Test (CTMT). Archives of Clinical Neuropsychology, 19, 703-708. http://acn.oxfordjournals.org/cgi/doi/10.1016/j.acn.2004.02.004 https://doi.org/10.1016/j.acn.2004.02.004

[19] Adegbite, A.B., Khan, M.I., Paine, K.W. and Tan, L.K. (1983) The Recurrence of Intracranial Meningiomas after Surgical Treatment. Journal of Neurosurgery, 58, 51-56. https://doi.org/10.3171/jns.1983.58.1.0051

[20] Tzourio-Mazoyer, N., Landeau, B., Papathanassiou, D., Crivello, F., Etard, O., Delcroix, N., et al. (2002) Automated Anatomical Labeling of Activations in SPM Using a Macroscopic Anatomical Parcellation of the MNI MRI Single-Subject Brain. NeuroImage, 15, 273-289. https://doi.org/10.1006/nimg.2001.0978

[21] Grieve, S.M., Williams, L.M., Paul, R.H., Clark, C.R. and Gordon, E. (2007) Cognitive aging, Executive Function, and Fractional Anisotropy: A Diffusion Tensor MR Imaging Study. American Journal of Neuroradiology, 28, 226-235.

[22] Fellgiebel, A., Schermuly, I., Gerhard, A., Keller, I., Albrecht, J., Weibrich, C., et al. (2008) Functional Relevant Loss of Long Association Fibre Tracts Integrity in Early Alzheimer's Disease. Neuropsychologia, 46, 1698-1706. https://doi.org/10.1016/j.neuropsychologia.2007.12.010

[23] Müller, M.J., Mazanek, M., Weibrich, C., Dellani, P.R., Stoeter, P. and Fellgiebel, A. (2006) Distribution Characteristics, Reproducibility, and Precision of Region of Interest-Based Hippocampal Diffusion Tensor Imaging Measures. American Journal of Neuroradiology, 27, 440-446.

[24] Provenzale, J.M., McGraw, P., Mhatre, P., Guo, A.C. and Delong, D. (2004) Peritumoral Brain Regions in Gliomas and Meningiomas: Investigation with Isotropic DiffusionWeighted MR Imaging and Diffusion-Tensor MR Imaging. Radiology, 232, 451-460. https://doi.org/10.1148/radiol.2322030959 
[25] Abdullah, K.G., Lubelski, D., Nucifora, P.G.P. and Brem, S. (2013) Use of Diffusion Tensor Imaging in Glioma Resection. Neurosurgical Focus, 34, E1.

http://www.ncbi.nlm.nih.gov/pubmed/23544405

https://doi.org/10.3171/2013.1.FOCUS12412

[26] Kono, K., Inoue, Y., Nakayama, K., Shakudo, M., Morino, M., Ohata, K., et al. (2001) The Role of Diffusion-Weighted Imaging in Patients with Brain Tumors. American Journal of Neuroradiology, 22, 1081-1088. http://www.ncbi.nlm.nih.gov/pubmed/11415902

[27] Bosch, B., Arenaza-Urquijo, E.M., Rami, L., Sala-Llonch, R., Junqué, C., Solé-Padullés, C., et al. (2012) Multiple DTI Index Analysis in Normal Aging, Amnestic MCI and AD. Relationship with Neuropsychological Performance. Neurobiology of Aging, 33, 61-74. https://doi.org/10.1016/j.neurobiolaging.2010.02.004

[28] Wang, J.H., Lv, P.Y., Wang, H.B., Li, Z.L., Li, N., Sun, Z.Y., et al. (2013) Diffusion Tensor Imaging Measures of Normal Appearing White Matter in Patients Who Are Aging, or Have Amnestic Mild Cognitive Impairment, or Alzheimer's Disease. Journal of Clinical neuroscience, 20, 1089-1094. https://doi.org/10.1016/j.jocn.2012.09.025

[29] Ivanco, T.L. and Greenough, W.T. (2000) Physiological Consequences of Morphologically Detectable Synaptic Plasticity: Potential Uses for Examining Recovery Following Damage. Neuropharmacology, 39, 765-776. https://doi.org/10.1016/S0028-3908(00)00004-6

[30] Grover, K.M., Bowyer, S.M., Rock, J., Rosenblum, M.L., Mason, K.M., Moran, J.E., et al. (2006) Retrospective Review of MEG Visual Evoked Hemifield Responses Prior to resecTion of Temporo-Parieto-Occipital Lesions. Journal of Neuro-Oncology, 77, 161-166. https://doi.org/10.1007/s11060-005-9014-Z

[31] Bavelier, D. and Neville, H.J. (2002) Cross-Modal Plasticity: Where and How? Nature Reviews Neuroscience, 3, 443-452.

Submit or recommend next manuscript to SCIRP and we will provide best service for you:

Accepting pre-submission inquiries through Email, Facebook, LinkedIn, Twitter, etc.

A wide selection of journals (inclusive of 9 subjects, more than 200 journals)

Providing 24-hour high-quality service

User-friendly online submission system

Fair and swift peer-review system

Efficient typesetting and proofreading procedure

Display of the result of downloads and visits, as well as the number of cited articles

Maximum dissemination of your research work

Submit your manuscript at: http://papersubmission.scirp.org/

Or contact jbise@scirp.org 